\title{
Structural Design Optimization-Numerical and Simulation Approaches
}

\section{Dr. Akbar M. Eslami, Elizabeth City State University}

Dr. Akbar Eslami is a professor and Engineering Technology coordinator in the Department of Technology at Elizabeth City State University. He received his Ph.D. in Mechanical Engineering from Old Dominion University. His research interests are in Computer Aided Manufacturing and Design, Reverse Engineering, Finite Element Analysis, and Design Optimization.

\section{Mr. Momen Nasri Mohammad, Elizabeth City State University}

Momen N. Mohammad is a graduate of Elizabeth City State University, acquiring a Bachelor Degree of Science in Engineering Technology, focus on Mechanics and Automation. Momen also minored in Mathematics, as he desired to be versatile and knowledgeable in the Engineering world. His research interest are in structural engineering, bridges, and aspires further education in those fields. 


\title{
Structural Design Optimization: Numerical and Simulation Approaches
}

\begin{abstract}
A structure with optimal mechanical properties such as weight and stiffness have a significant positive impact on enhancing performance and cost efficiency. A very common structural optimization application problem arises in finding minimal weight design with constraints on stress and deflection. Real world applications, such as designing structural components of trusses, bridges, cars, aircraft, and spacecraft can be formulated and solved as engineering optimization problems. The goal of this project is to utilize computational methods including numerical and simulation analyses to find various optimal values and study the behavior of the structure. The MATLAB optimization solver is used to find optimum values numerically without violating constraints. The optimal values are, in turn, used as design parameters in the SolidWorks simulation software for creating a Computer Aided Design (CAD) model and virtually simulating a CAD model of the structure to ensure that the structure can withstand realworld physical behavior. This project will have positive impacts on training and educating students in areas of design optimization, computational, and simulation methods.
\end{abstract}

\section{Introduction}

According to the Oxford Dictionary, the word "optimize" in a general setting can be defined as making the best or most effective use of a situation, opportunity, or resource without violating any constraints. Modern optimization methods were pioneered by Courant's paper [1] on penalty functions, Dantzig's paper [2] on the simplex method for linear programming, and Karush, Kuhn, and Tucker, who derived the KKT optimality conditions for constrained problems [3]. The use of nonlinear optimization techniques in structural design was pioneered by Schmit [4].

Today, many engineering problems involved in design optimization are subjected to constraints. Design optimization plays a key role in design and has a wide variety of applications in realworld problems. Applications include increasing stiffness or reducing the weight of the structural components in spacecraft, aircraft, automobiles, and other structures without sacrificing structural integrity.

The three examples in this paper can be used as a module/modules to teach applications of optimization and simulation in an undergraduate Finite Element Analysis course. The modules can be presented to students with a slight knowledge of optimization theory and MATLAB software. This project offers students the opportunity to gain valuable computational skills in areas of design optimization, CAD, and simulation. Also, students will learn to formulate the optimization problem and choose appropriate MATLAB optimization solvers to yield optimal values and validate the optimal results by virtually simulating the CAD model.

\section{Methodology}

The concept of optimization has four elements: design variables, objective function, constraints, and design space. Design variables include numerical quantities that will vary throughout the optimization process. Examples of design variables include, but are not limited to, size, weight, and geometry. The objective function is a mathematical equation that represents the design 
variables that will be optimized. Constraints are restrictions that define limitations of physical quantities in a specific design. The design space is the domain defined by the design variables.

In this project, three structure models are analyzed to obtain optimal design variables, and structures are simulated to assess the accuracy of the optimal results. First, the objective function and constraints are defined, and the appropriate MATLAB optimization solver is used to minimize objective function while satisfying constraints. Prior to simulation, the optimal values of the design variable yielded from the MATLAB solver are utilized to model the structure in the SolidWorks CAD software. The model is, in turn, simulated to virtually test and analyze the structure to ensure that it can withstand the loading condition and doesn't violate constraints. Three problems are presented as follows:

\section{Truss Problem Statement:}

As shown in Figure1, a steel truss has been designed with no maximum load capacity given. The four concentrated loads are applied to four joints located at the top of the truss, including points $\mathrm{B}$ and $\mathrm{E}$. Maximum permissible stresses in members $\mathrm{AB}$ and $\mathrm{BC}$ are $71.2 \mathrm{MPa}$ and $18.5 \mathrm{MPa}$, respectively, and the permissible vertical deflection at point $\mathrm{C}$ is $3 \mathrm{~mm}$. All members have an equal cross section area and length.

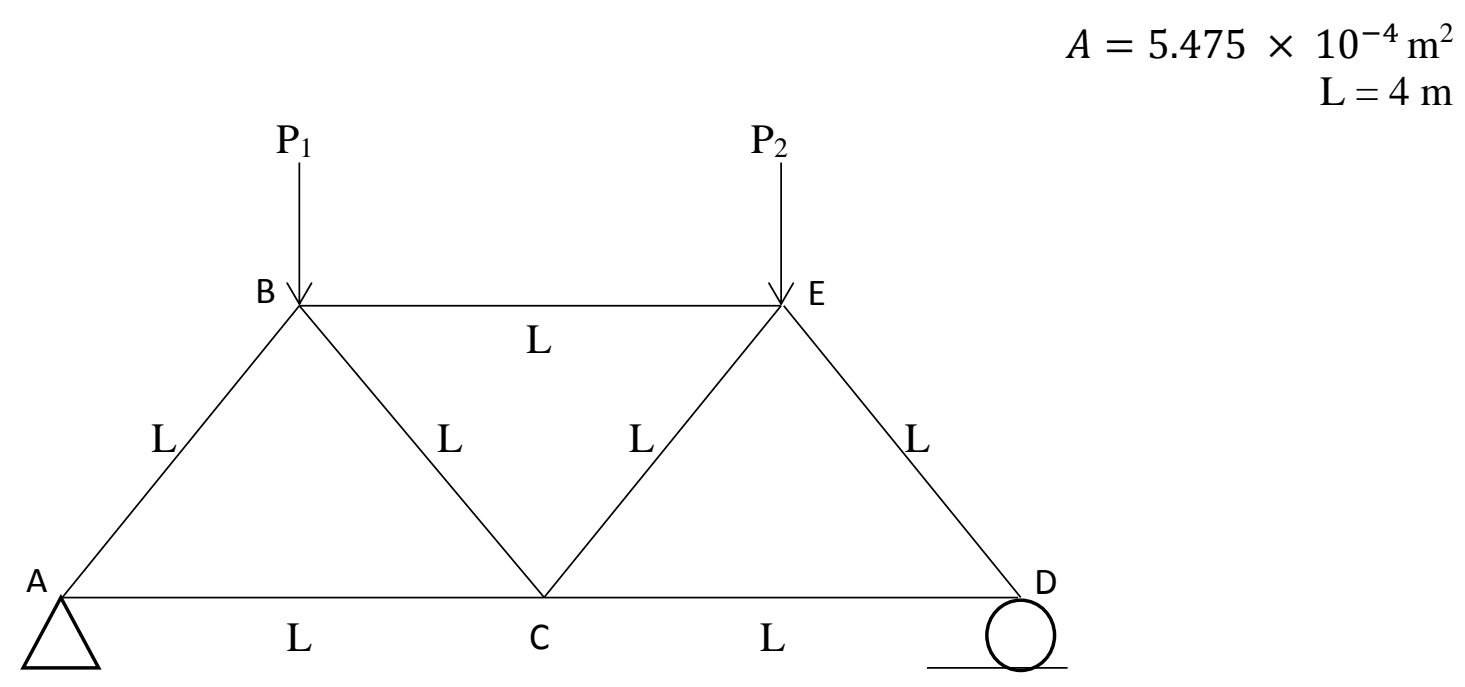

Fig. 1: Truss Problem

The optimization problem to maximize the load capacity is given:

Maximize: $\quad P_{1}+P_{2}$

Subject to: $\quad 3 P_{1}+P_{2} \leq 135.1 \mathrm{kN} ;-3 P_{1}-P_{2} \leq 135.1 \mathrm{kN} ; P_{1}-P_{2} \leq 35.1 \mathrm{kN}$;

$-P_{1}+P_{2} \leq 35.1 \mathrm{kN} ; P_{1}+P_{2} \leq 85.1 \mathrm{kN} ;-P_{1}-P_{2} \leq 85.1 \mathrm{kN}$;

$P_{i} \geq 0 \quad i=1,2$. 


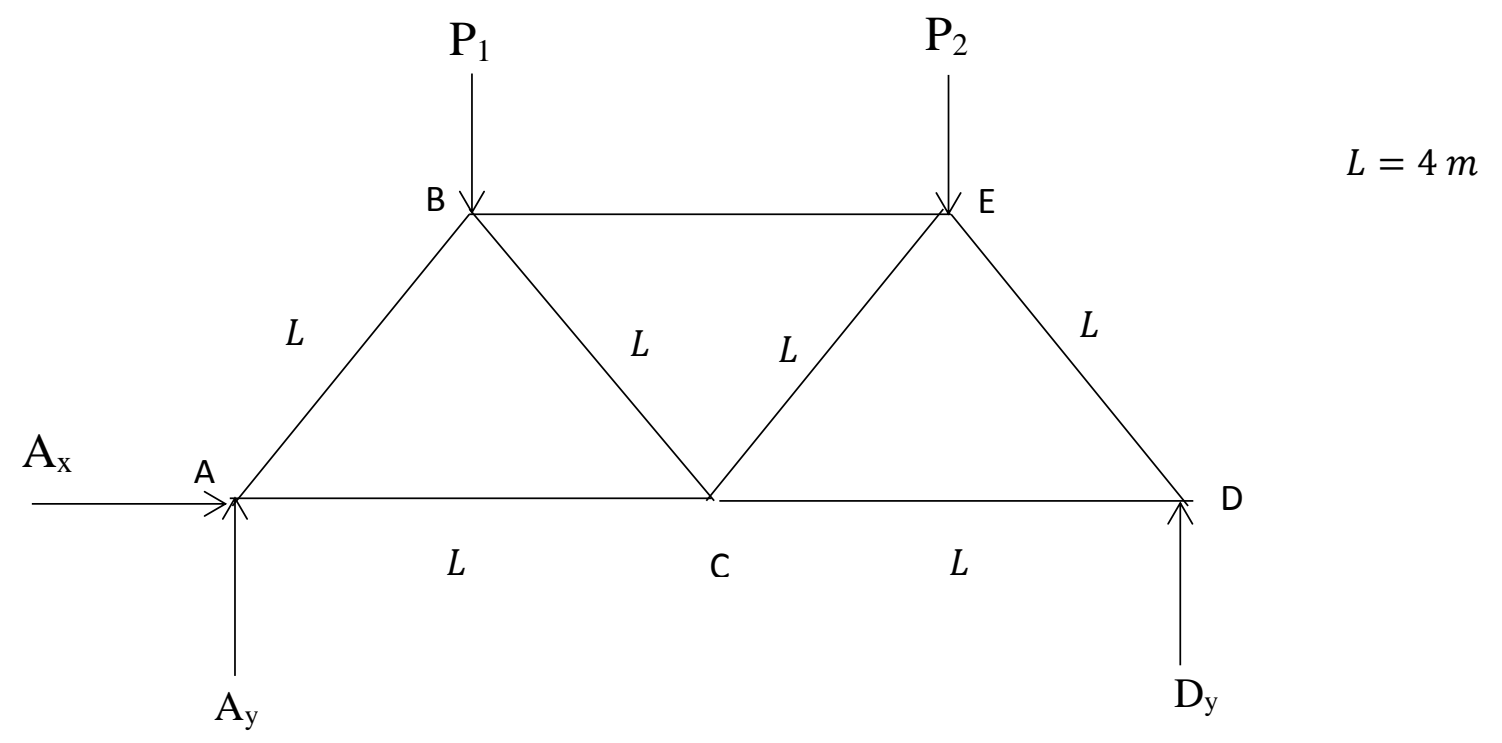

Fig. 2: Free Body Diagram

\section{$\underline{\text { Stress in Member AB }}$}

$$
\begin{array}{lcr}
\begin{array}{l}
\sum F_{x}=0 \\
A_{x}=0
\end{array} & \begin{array}{c}
\sum M_{A}=0 \\
A_{y}+D_{y}-P_{1}-P_{2}=0
\end{array} \\
A_{y}+D_{y}=P_{1}+P_{2} & -2 P_{1}-6 P_{2}+8 D_{y}=0 \\
A_{y}+\frac{3 P_{2}+P_{1}}{4}=P_{1}+P_{2} & D_{y}=\frac{3 P_{2}+P_{1}}{4} \\
\text { At Joint A: } & A_{y}=P_{1}+P_{2}-\left(\frac{3 P_{2}+P_{1}}{4}\right) & A_{y}=\frac{3 P_{1}+P_{2}}{4} \\
A_{x} & A B \frac{\sqrt{3}}{2} &
\end{array}
$$
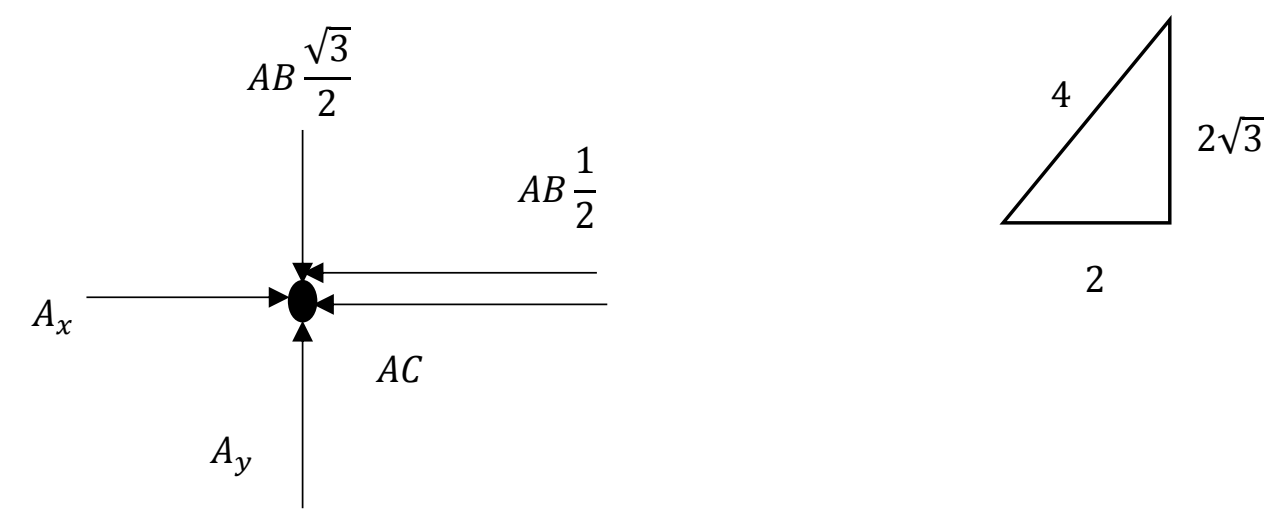
$\sum F_{y}=0$

$A_{y}-A B \frac{\sqrt{3}}{2}=0$

$\frac{3 P_{1}+P_{2}}{4}-A B \frac{\sqrt{3}}{2}=0$

$A B=\frac{3 P_{1}+P_{2}}{2 \sqrt{3}}$

Stress in member AB:

$$
\frac{A B}{\text { Area }}=\frac{3 P_{1}+P_{2}}{2 \sqrt{3} \times \text { Area }}
$$

Stress in member BC:

$$
\frac{B C}{\text { Area }}=\frac{P_{1}-P_{2}}{2 \sqrt{3} \times \text { Area }}
$$

Stress in member $\mathrm{AB}$ is equal to Force AB/Area which should be less than 71.2 MPa. Stress in member $\mathrm{BC}$ is equal to Force BC/Area which should be less than 18.5 MPa. The constraint equations were obtained after substituting the area value in stress equations.

\section{a. MATLAB Results}

The linprog solver in the MATLAB code is used to minimize the objective function since the problem is linear. Note that maximizing the objective function is equivalent to minimizing the objective function multiplied by $(-1)$. Then to properly formulate the optimization problem, the objective function is formulated as:

Minimize $-P_{1}-P_{2}$

The MATLAB format of the objective function and constraints is listed as

$>\mathrm{f}=[-1-1]$

$>\mathrm{A}=[31 ;-3-1 ; 1-1 ;-11 ; 11 ;-1-1]$

$>\mathrm{b}=[135.1 ; 135.1 ; 35.1 ; 35.1 ; 85.1 ; 85.1]$

$>$ Aeq $=[]$

$>$ beq $=[]$

$>\mathrm{lb}=\left[\begin{array}{ll}0 & 0\end{array}\right]$

$>\mathrm{ub}=[]$

$>$ > $\mathrm{X}, \mathrm{Z}]=$ linprog (f,A,b,Aeq,beq,lb,ub)

The optimal solution yielded the values of 25 $\mathrm{KN}$ and $60.1 \mathrm{KN}$ for $P_{1}$ and $P_{2}$, respectively.

\section{b. $\underline{\text { CAD Model and Simulation }}$}

The optimal values of $P_{1}$ and $P_{2}$ are used in the SolidWorks simulation software to validate the allowable stresses in members of $A B$ and $B C$ as well as deflection in joint $C$ the truss. The CAD model is given in Figure 3. 


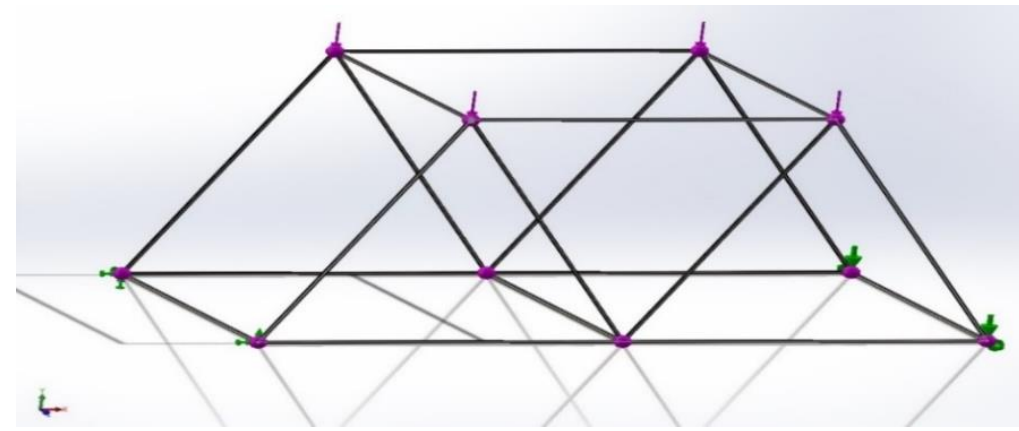

Fig. 3: SolidWorks CAD Model of Truss with Optimal Loads

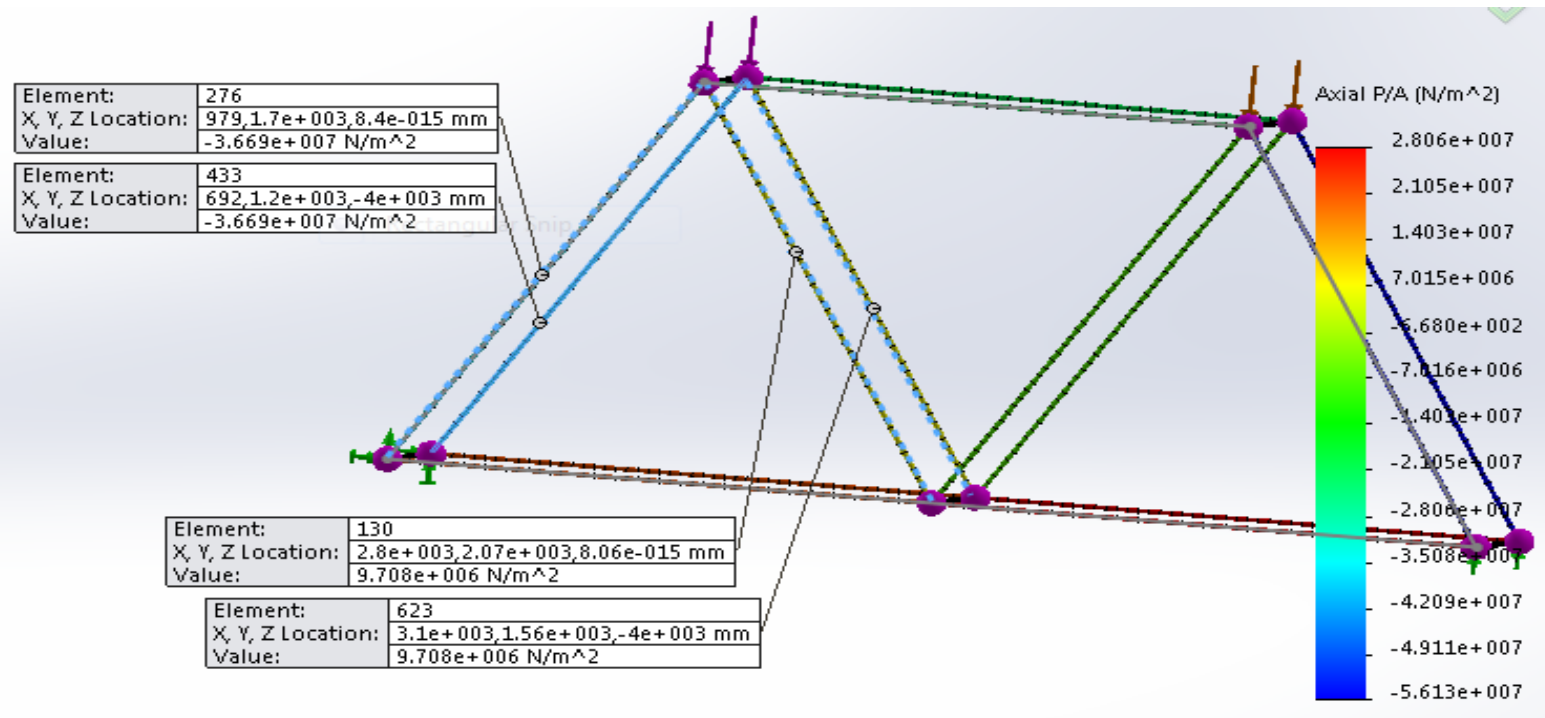

Fig.4: Stress Analysis on Truss

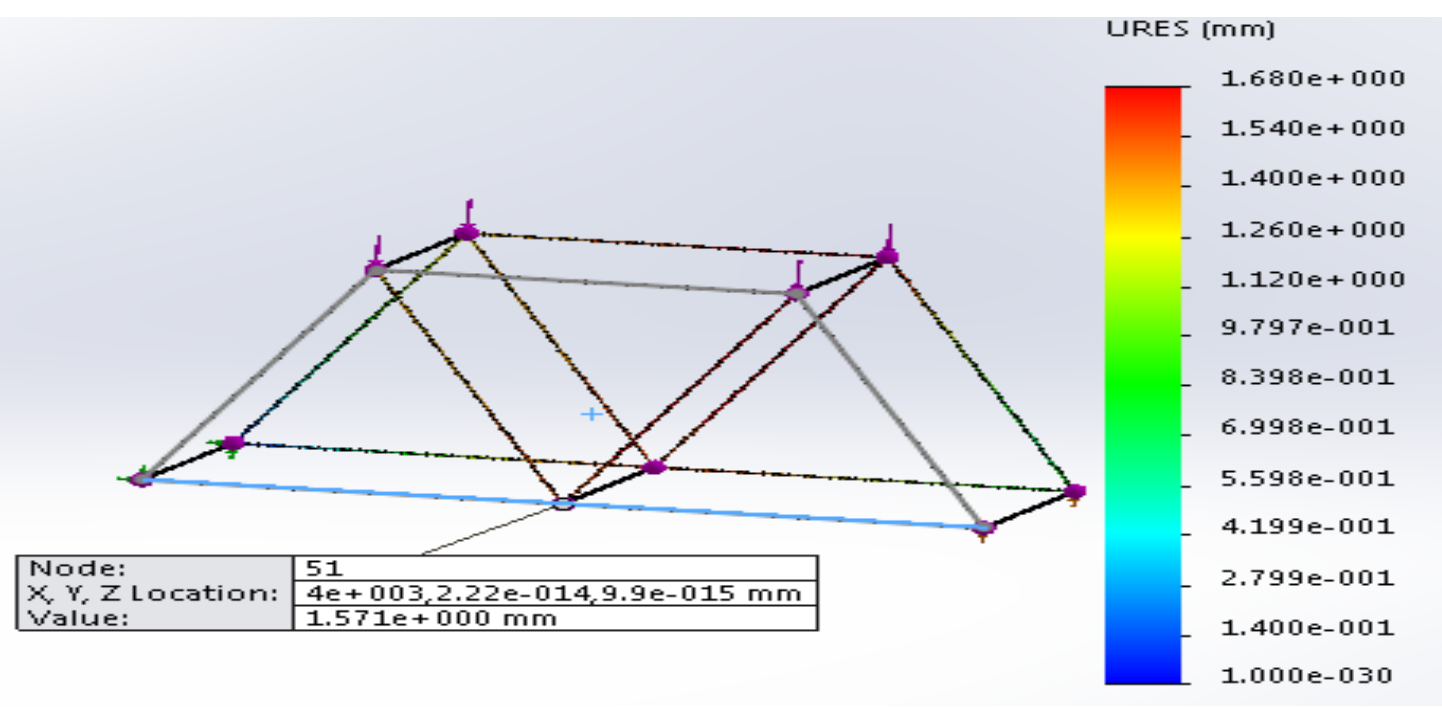

Fig.5: Displacement Analysis 
The Figure 4 stress simulation result verifies that the stresses in members $\mathrm{AB}$ and $\mathrm{BC}$ are lower than 71.2 $\mathrm{MPa}$ and 18.5 $\mathrm{MPa}$, respectively. Also, Figure 5 shows that deflection in joint $\mathrm{C}$ is lower than the allowable $3 \mathrm{~mm}$ deflection.

\section{Welded Beam Problem Statement:}

As depicted in Figure 6, a cantilever beam is welded onto a column. The welding area has two segments, each with length $l$ and width $b$ sizes. The length of beam $L=14$ inches is subjected to a load $F=6000 \mathrm{lbs}$.
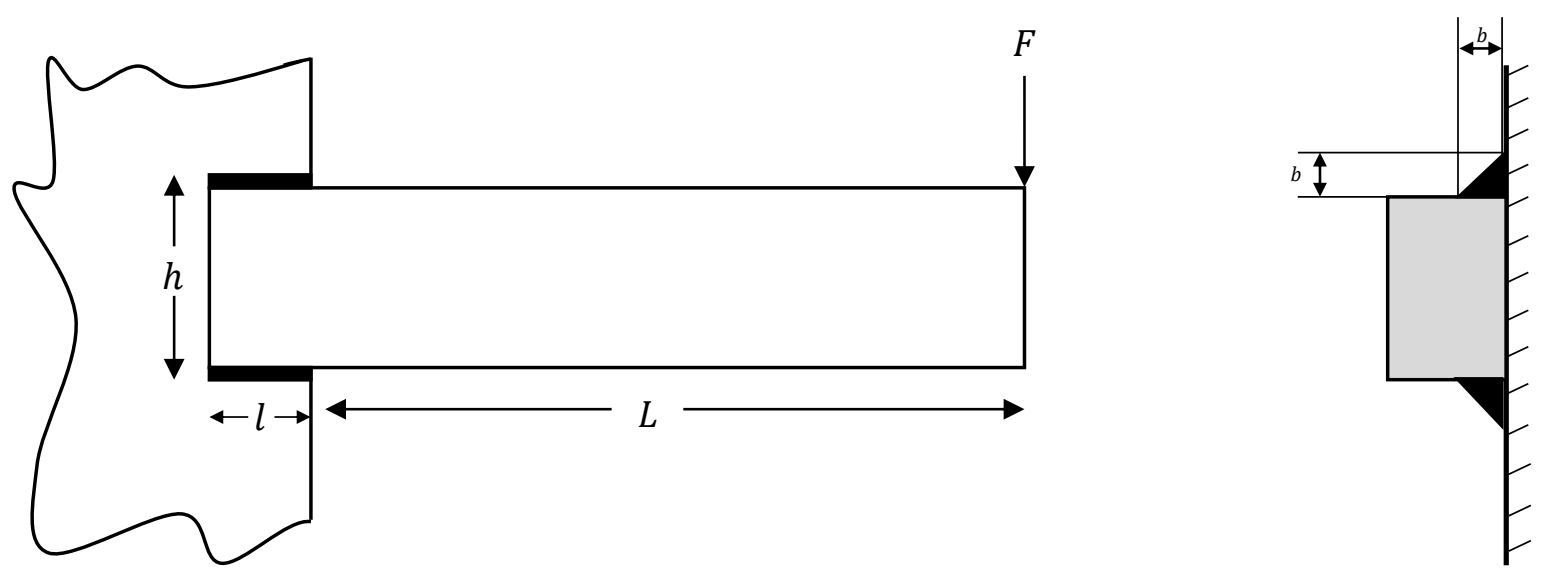

Fig. 6: Cantilever Beam

The objective of this problem is to minimize welding volume subjected to a limited shear stress on the welded area.

Objective Function: $f=2 b^{2} l$

Constraints: $\quad \tau \leq 30,000$ psi; $0.25 \leq b \leq 1$ in; $2 \leq l \leq 3$ in; $h=3$ in

The expression for $\tau$ is given as:

$\tau_{y}=\frac{F}{b l \sqrt{2}}$

$\tau_{t}=\frac{6 F(L+0.5 l) \sqrt{\left(h^{2}+l^{2}\right)}}{\sqrt{2 b l\left(l^{2}+3 h^{2}\right)}}$

$\tau=\sqrt{\tau_{y}^{2}+2 \tau_{y} \tau_{t} \cos \theta+\tau_{t}^{2}} \quad ; \cos \theta=\frac{l}{\sqrt{\left(h^{2}+l^{2}\right)}}$

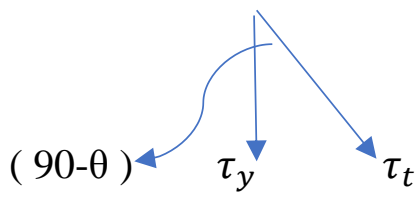

\section{a. MATLAB Results}

The MATLAB code to generate the optimal results is given as follows:

In MATLAB coding, $\mathrm{x}(1)$ and $\mathrm{x}(2)$ are $b$ and $l$, respectively.

function $\mathrm{f}=\operatorname{objecfun}(\mathrm{x})$

$\mathrm{f}=2^{*}\left(\mathrm{x}(1)^{\wedge} 2\right)^{*} \mathrm{x}(2)$;

function $[\mathrm{c}, \mathrm{ceq}]=$ nonlconstr $(\mathrm{x})$

$\mathrm{y}(1)=6000 /(\mathrm{x}(1) * \mathrm{x}(2) * 1.41)$; 
$\mathrm{y}(2)=\left(36000 *(14+0.5 * \mathrm{x}(2)) * \operatorname{sqrt}\left(9+\mathrm{x}(2)^{\wedge} 2\right)\right) /\left(1.41 * \mathrm{x}(1)^{*}\left(\mathrm{x}(2)^{\wedge} 3+27 * \mathrm{x}(2)\right)\right)$;

$\mathrm{c}=\left[\operatorname{sqrt}\left(\mathrm{y}(1)^{\wedge} 2+2^{*} \mathrm{y}(1)^{*} \mathrm{y}(2)^{*} \mathrm{x}(2) /\left(9+\mathrm{x}(2)^{\wedge} 2\right)+\mathrm{y}(2)^{\wedge} 2\right)-30000\right]$;

ceq $=[]$

The MATLAB software yielded the optimal values for $b=0.528$ in and $l=3$ in.

\section{b. CAD Model and Simulation}

The optimal values for $b$ and $l$ are utilized in the CAD model and SolidWorks simulation software is used to validate the allowable shear stress on the welded area. The CAD model is given in Figure 7.

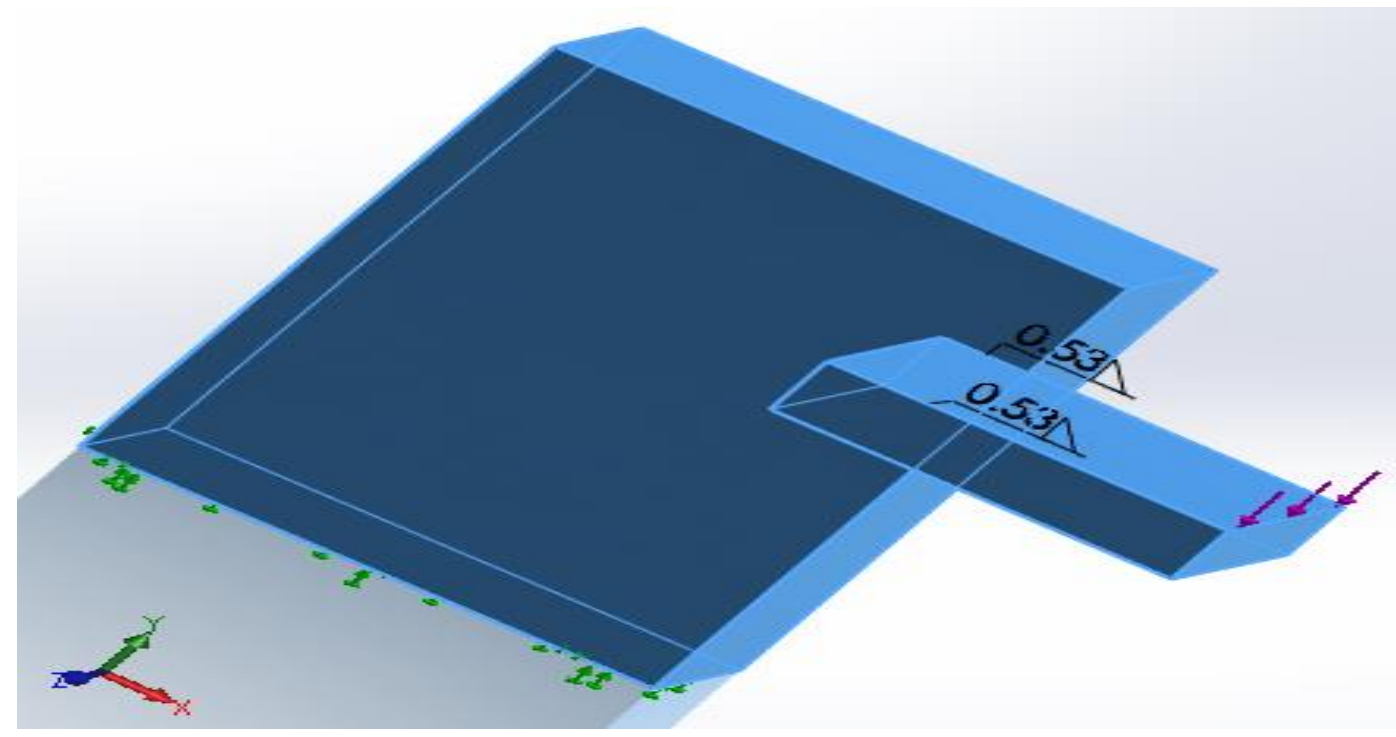

Fig.7: SolidWorks CAD Model with Optimal Welded Sizes for Welded Beam
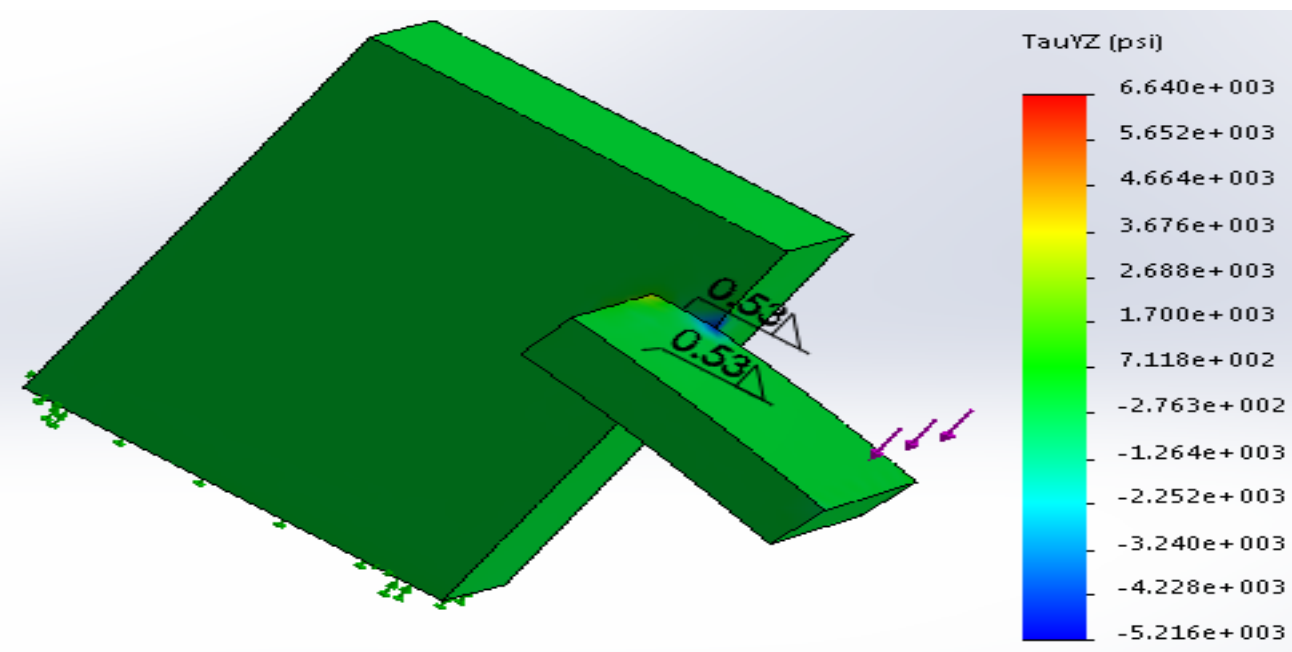

Fig.8: Welded Beam $\tau_{y z}$ Shear Stress Results 


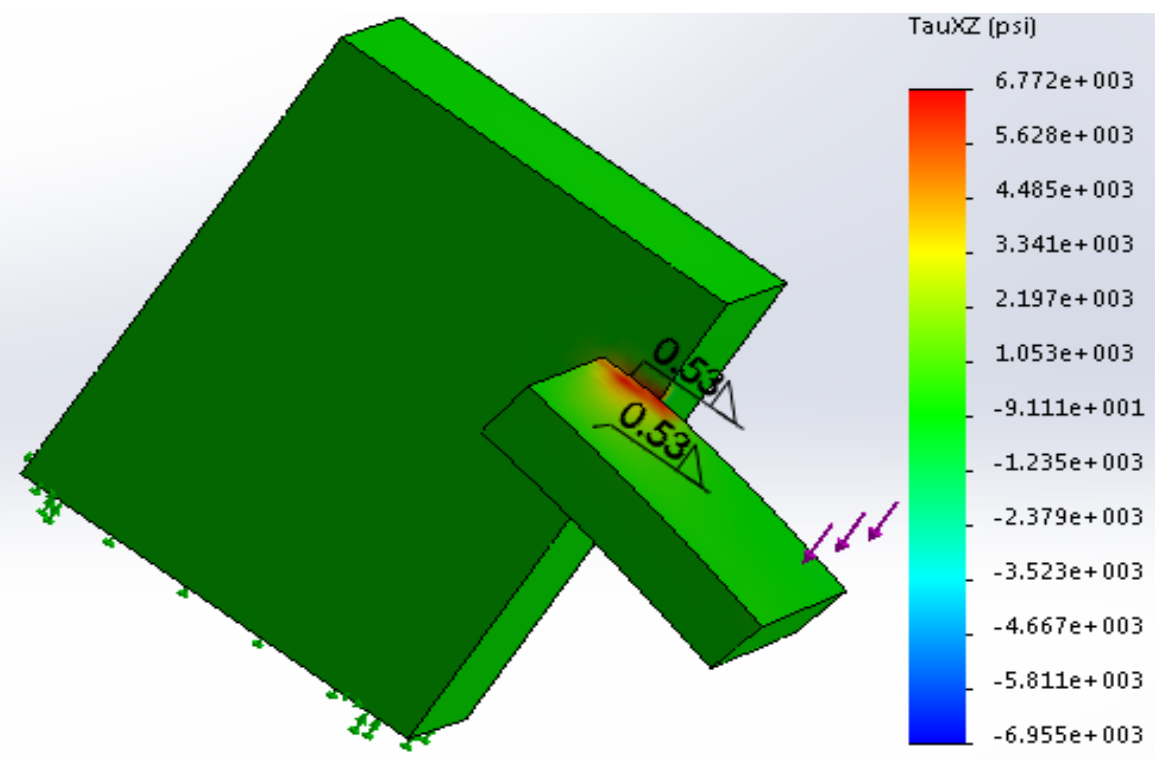

Fig. 9: Welded Beam $\tau_{x z}$ Shear Stress Results

The Figures 8 and 9 resulted from simulation software and validate that the $\tau$ shear stress on the welded area is lower than 30,000 psi.

\section{Two-Bar Truss Problem Statement:}

The design of a two-bar truss is given in Figure 10. The load $\mathrm{P}$ is applied at node $\mathrm{A}$ which causes member $\mathrm{AB}$ to be in tension and member $\mathrm{AC}$ to be in compression. A simple minimum weight design problem here would be to minimize the weight of the truss subjected to: (i) ensuring that each member does not yield, and (ii) the member AC, which is in compression, does not buckle.

A design vector $x=\left[A_{1}, A_{2}, H\right]$ is defined where $A_{1}$ and $A_{2}$ are area cross-sections and $H$ is the vertical distance between two bars. Thus, the problem may be stated as:

$E_{\text {steel }}=30 \times 10^{6} \mathrm{psi}, \gamma=0.2836 \mathrm{lb} / \mathrm{in}^{3}, S_{y}=36,260 \mathrm{psi}, F_{\mathrm{s}}=1.5, P=15,000 \mathrm{lb}, \mathrm{L}=5 \mathrm{in}$

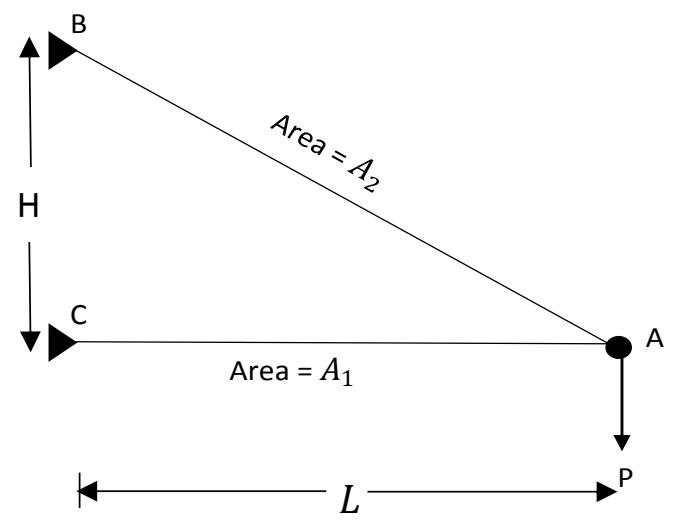

Fig. 10: Two-Bar Truss 
Minimize: $\quad \gamma A_{1} L+\gamma A_{2} \sqrt{\left(L^{2}+H^{2}\right)}$

Subject to: $\quad \frac{P}{A_{2} \sin \theta} \leq S_{y} / F_{s} ; \frac{P}{A_{1} \tan \theta} \leq S_{y} / F_{s} ; \frac{P}{\tan \theta} \leq \frac{\pi^{2} E I_{1}}{L^{2} F_{s}}$

$A_{1}, A_{2}, H \geq 0$

\section{a. MATLAB Solutions}

The objective function and constraints given in the problem statement are utilized in the

MATLAB software. In MATLAB coding, $A_{1}, A_{2}$, and $H$ are $\mathrm{x}(1), \mathrm{x}(2)$, and $\mathrm{x}(3)$ respectively. For a circular cross-section $I_{1}=\frac{A_{1}{ }^{2}}{4 \pi}$ and substituting for $\theta$, in joint $\mathrm{A}$, in terms of $L$ and $H$, the optimization equations yield:

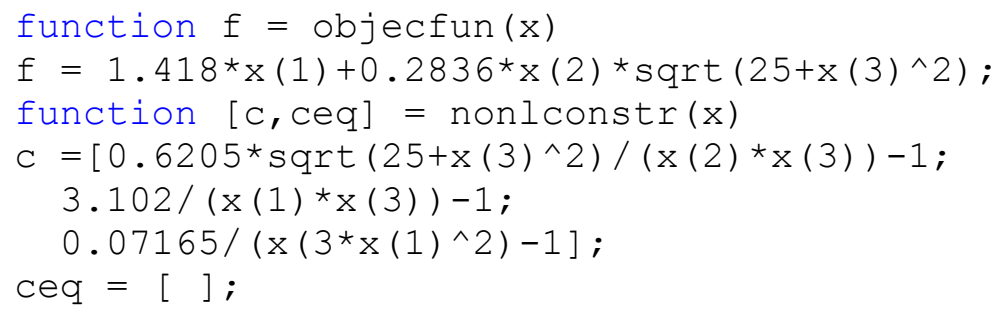

The MATLAB code yielded the optimal values of $A_{1}=0.653 \mathrm{in}^{2}, A_{2}=0.8 \mathrm{in}$, and $H=5 \mathrm{in}$.

\section{b. CAD Model and Simulation}

The optimal values obtained from the MATLAB code are utilized in the CAD model and SolidWorks simulation software is used to validate allowable stresses. The CAD model of the Two-Bar truss is given in Figure 11. Note that the size and geometry of the Two-Bar truss is 1.5 sch 40 circular pipe that has area approximately $0.8 \mathrm{in}^{2}$.

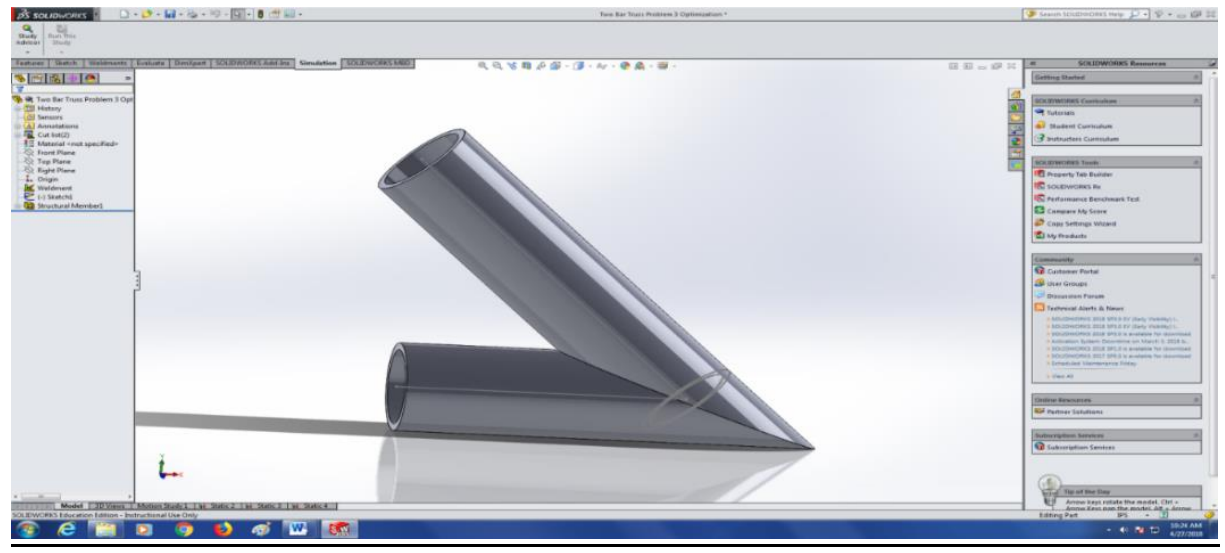

Fig. 11: SolidWorks CAD Model of the Two-Bar Truss with Optimal Values 


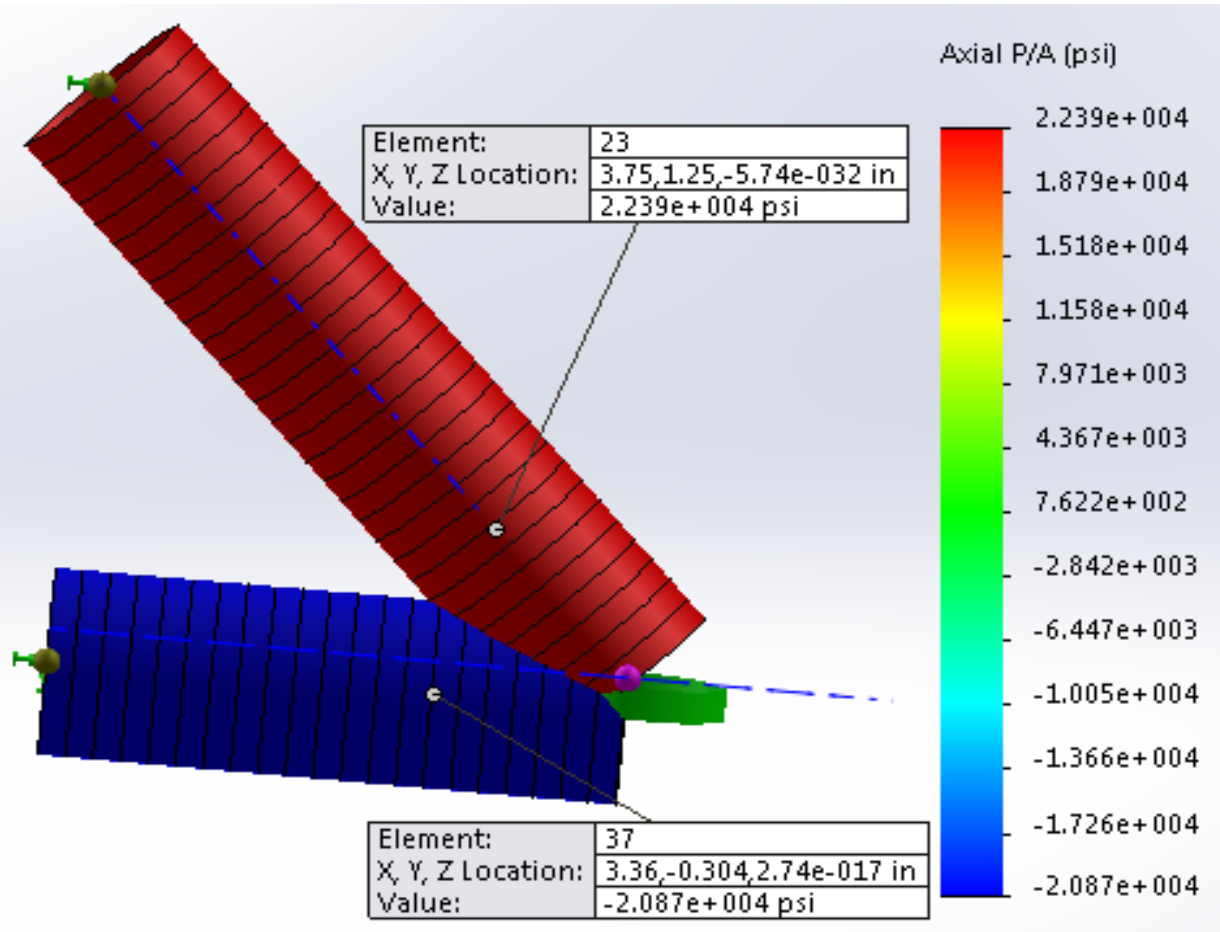

Fig. 12: Two-Bar Truss Axial Stress Simulation Results

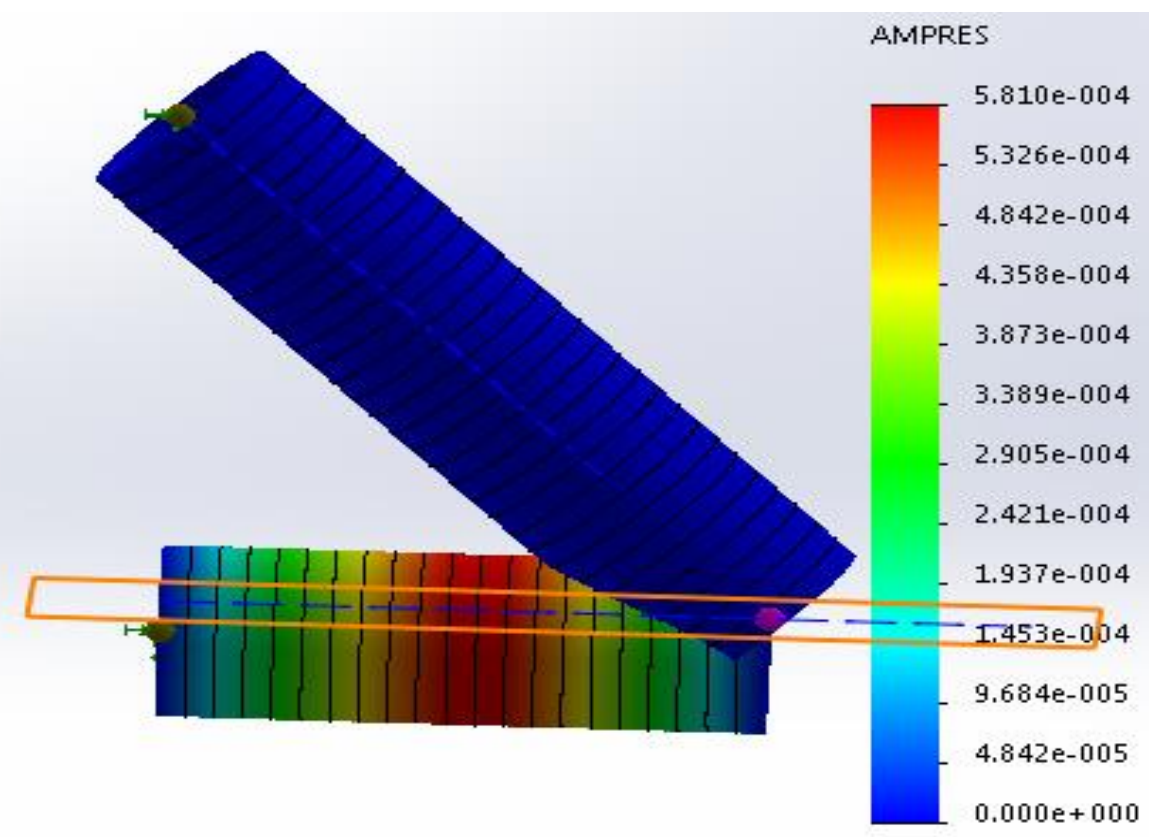

Fig.13: Amplitude Results from Buckling 
Figure 12 resulted from the simulation and verifies that axial stress is lower than the allowable axial stress 24173 psi which obtained by substituting the value of $S_{y} / F_{S}$ in stress equations. Figure 13 shows amplitude of deflection resulted from buckling which are very close to zero.

\section{Assessment of the Project}

This project requires students to utilize their knowledge and skills as well as demonstrate the level of learning stated on the ABET-ETAC student learning outcomes. The project can be used as a direct assessment method to validate student outcomes and evidence of what students have learned from the curriculum. This project is used to measure students' mastery in the simulation subject area. A survey was designed to get feedback from students in the Finite Element Analysis / Engineering Software Application course. This survey allows the student to rate the student's achievements in terms of how successful he/she was in achieving the stated student outcomes and rates the quality of modules and instruction.

\section{Conclusion and Future Research}

Computational simulation is utilized in conjunction with the MATLAB numerical solution to validate the optimal results. The computational simulation not only supports the numerical results, but it can also be extended to more complicated designs. There are some anticipated challenges with the CAD and simulation modeling portions. Ensuring the model has the correct input of boundary conditions, force location and distribution, and material properties is critical to the success of the project.

More examples on structural design optimization, such as optimizing the size of beams and pressure vessels, can be included in future work. This can be completed by using more advanced and complex problems. The use of supercomputing clusters will alleviate some computational time concerns. Also, formulating objective functions with constraints can be investigated as well.

\section{References}

1. Courant, R. Variational methods for the solution of problems of equilibrium and vibrations, Bulletin of the American Mathematical Society, 49,1-23,1943.

2. Dantzig, G.B., Maximization of a Linear Function of Variables Subject to Linear Inequalities, in T.C. Koopmans (Ed.) Activity Analysis of Production and Allocation, Wiley, New York,1951, Chapter 21.

3. Kuhn, H.W. and Tucker, A.W., Non-linear programming, Proceeding of the second Berkeley symposium on mathematical statistics and probability, University of California press, Berkeley,1951, pp.481-493.

4. Schmit, L.A., Structural design by systematic synthesis, Proceeding of the $2^{\text {nd }}$ conference on electronic computation, ASCE, New York, pp.105-122, 1960. 